\title{
Neutrophil-lymphocyte ratio is associated with arterial stiffness in patients with peritoneal dialysis
}

\author{
Kedan Cai, Qun Luo*, Beixia Zhu, Lina Han, Dan Wu, Zhiwei Dai and Kaiyue Wang
}

\begin{abstract}
Background: Patients with peritoneal dialysis are in the persistent inflammation state and have elevated arterial stiffness. Neutrophil-lymphocyte ratio(NLR) is a new inflammatory marker in renal and cardiac disorders. Brachial-ankle pulse wave velocity (baPMV) is a non-invasive measurement, which is widely used as a surrogate marker of arterial stiffness. However, there is little evidence to show an association between NLR and baPW in patients with peritoneal dialysis. The aim of this cross-section study was to investigate the relationship between NLR and arterial stiffness measured by baPWV in patients with peritoneal dialysis.

Methods: In this cross-section study, 101 patients with peritoneal dialysis were enrolled from January 2014 to June 2015. According to average baPWW level $(1847.54 \mathrm{~cm} / \mathrm{s})$, the patients were categorized into two groups, low group and high group. baPWW, which reflects arterial stiffness, was calculated using the single-point method. Clinical data were collected in details. NLR was calculated using complete blood count. Associations between NLR and baPW were assessed using Pearson's correlation and linear regression analysis.

Results: The NLR was significantly lower in the low baPW group than in the high baPWW group $(p=0.03)$. There were positive correlations between baPW and neutrophil count $(r=0.24, p=0.01)$ and NRL $(r=0.43, P<0.01)$, and there was a negative correlation between baPW and lymphocyte count $(r=-0.23, p=0.01)$. In addition, albumin, phosphorous and intact parathyroid hormone showed negative correlations with baPWV $(r=-0.32, p<0.01 ; r=-0.28, p<0.01 ; r=-0.25$, $p=0.01$, respectively). Age and hsCRP showed positive correlations with baPW $(r=0.47, p<0.01 ; r=0.25, p=0.01)$. In multivariate analysis, NLR independently correlated with baPW in patients with peritoneal dialysis $(\beta=0.33, p<0.01)$, even after adjustment for various confounders.
\end{abstract}

Conclusion: Our study suggests that NLR was an independently associated with arterial stiffness in patients with peritoneal dialysis. However, further prospective studies are needed to confirm cause-and-effect relationship between NLR and baPWV, and to investigate whether anti-inflammatory treatment could improve arterial stiffness in patients with peritoneal dialysis.

Keywords: Neutrophil-lymphocyte ratio, Inflammation, Arterial stiffness, Brachial-ankle pulse wave velocity, Peritoneal dialysis

\footnotetext{
* Correspondence: luoqun@nbu.edu.cn

Department of Nephrology, Ningbo No. 2 Hospital, Ningbo University School of Medicine, Zhejiang, China
} 


\section{Background}

In 2014, 55,373 patients received peritoneal dialysis (PD) in China. Cardiovascular disease is the first etiology of mortality and morbidity, accounting for nearly $60 \%$ of all deaths in the patients with PD [1]. Microinflammation is a key component of the malnutrition- inflammationatherosclerosis and calcification syndrome (MIAC syndrome), which is associated with increased risk of cardiovascular disease in patients with $P D$.

Recently, neutrophil-to-lymphocyte ratio (NLR), calculated as a ratio of neutrophil to lymphocyte in peripheral blood, is regarded a readily available indicator for the severity and extension of systemic inflammation and atherosclerosis in renal and cardiac disorders [2, 3].

Elevated arterial stiffness is an early marker of systemic atherosclerosis, which has been shown to be a powerful independent predictor of cardiovascular events and all-cause mortality in chronic kidney disease(CKD) [4]. Pulse wave velocity (PWV) is a non-invasive measurement, which is widely used as a surrogate marker of arterial stiffness [5]. Brachial-ankle PWV (baPWV) correlates with the gold standard measurement, carotidfemoral PWV [6]. Increasing studies demonstrated that elevated baPWV is associated with increased risk of renal disease and cardiovascular diseases, as well as increased total mortality $[7,8]$.

However, there is little evidence to show an association between NLR and baPWV in patients with PD. Therefore, we aimed to evaluate the relationship between NLR and baPWV in patients with PD.

\section{Methods}

\section{Patients}

Patients with over one-month PD were included in this cross-section study from January 2014 to June 2015 in Ningbo No.2 Hospital. The number of cases during the study period determined the sample size. It has adhered to the STROBE guidelines for observational studies. According to average baPWV level $(1847.54 \mathrm{~cm} / \mathrm{s})$, the patients were categorized into two groups, low baPWV and high baPWV. Patients with active infection, severe liver dysfunction, malignancy, hematological diseases were excluded. The flow diagram is shown in Fig. 1. Patients were dialyzed with dextrose peritoneal dialysate produced by Baxter Healthcare (Guangzhou, China). This study was approved by the Ethics Committee of Ningbo No.2 Hospital.

\section{Demographic data and biochemical measurements}

The demographic data included age, gender, etiology of end-stage renal disease, prevalence of diabetes, the dialysis duration, current smoking status, and atherosclerotic vascular disease (AVD) history. Physical examination included blood pressure, height, and weight. Blood sample was drawn in the morning following a fasting period of over $8 \mathrm{~h}$. 24-hours ultrafiltration volume, and urine output volume were recorded. Biochemical measurements, using standard laboratory techniques in the same laboratory center, included blood cell counts, neutrophic counts, lymphocyte counts, haemoglobin, platelets, calcium, phosphorus, intact parathyroid hormone (iPTH), creatinine, urea nitrogen, total cholesterol, triglycerides, albumin and highly sensitive C-reactive protein (hsCRP). Complete

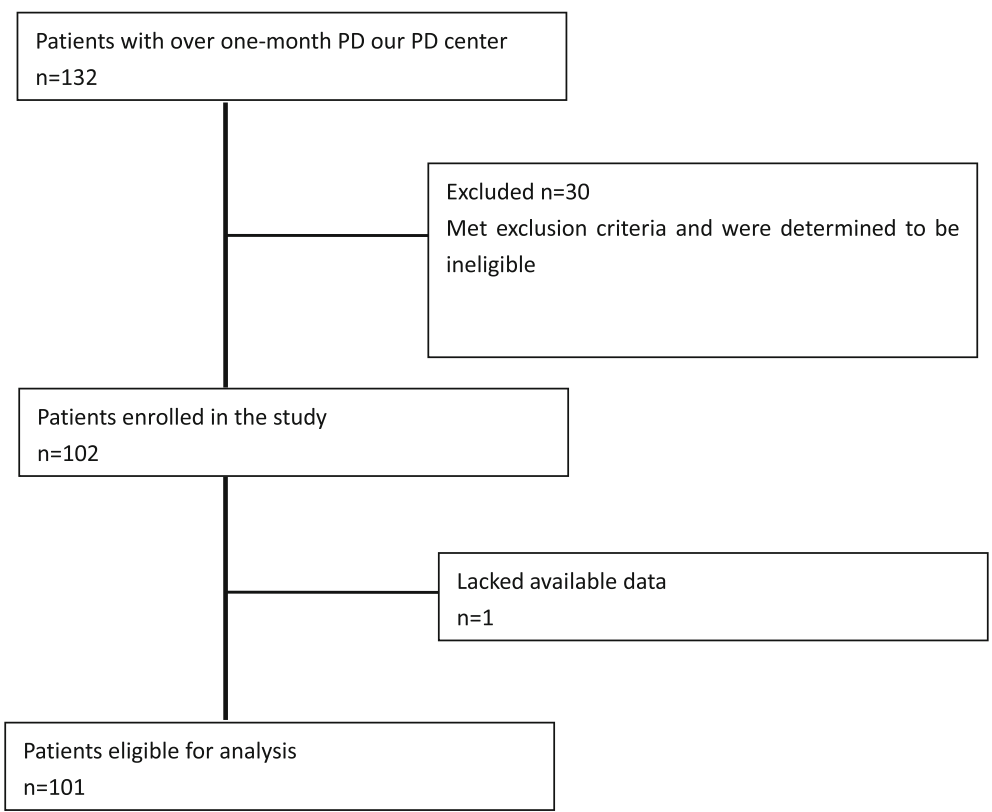

Fig 1. Patient selection scheme 
blood counts were analysed using Sysmex XT-2000i (Sysmex Corporation). Serum calcium, phosphate, renal profiles, albumin hsCRP, and lipid profiles were measured using ADVIA 2400 Chemistry System(Siemens Healthcare
Diagnostics). PTH levels were determined using ADVIA Centaur XP (Siemens Healthcare Diagnostics). All of these data were collected from Hospital Information System of Ningbo No.2 Hospital.

Table 1 Baseline demographic and clinical characteristics of PD patients stratified by average baPWV level

\begin{tabular}{|c|c|c|c|c|}
\hline \multirow[t]{2}{*}{ Variables } & \multicolumn{4}{|l|}{ PWV } \\
\hline & $\begin{array}{l}\text { Low } \\
(n=58)\end{array}$ & $\begin{array}{l}\text { High } \\
(n=43)\end{array}$ & $\mathrm{T} / \mathrm{u} / \mathrm{x}^{2}$ & $P$-value \\
\hline Age (year) & $48.60 \pm 14.20$ & $63.60 \pm 12.65$ & -5.49 & $<0.01^{*}$ \\
\hline Gender (male, \%) & $30(51.72)$ & $30(69.76)$ & 3.33 & 0.06 \\
\hline Body mass index $\left(\mathrm{kg} / \mathrm{m}^{2}\right)$ & $21.16 \pm 3.03$ & $20.81 \pm 2.83$ & 0.59 & 0.55 \\
\hline Peritoneal dialysis duration (months) & & & -0.05 & 0.95 \\
\hline Median & 16.00 & 16.00 & & \\
\hline Interquartile range & $4.75,45.00$ & $7.00,46.00$ & & \\
\hline Diabetes (\%) & $6(10.34)$ & 13(30.23) & 6.39 & $0.01^{*}$ \\
\hline Etiology of ESRD & & & 5.59 & 0.06 \\
\hline Chronic glomerular nephritis (\%) & $44(75.86)$ & 23(53.48) & & \\
\hline Diabetic kidney disease (\%) & $5(5.17)$ & $8(18.60)$ & & \\
\hline Others(\%) & $9(15.51)$ & 12(27.90) & & \\
\hline current smoking status(\%) & $5(8.62)$ & 6(13.95) & 0.72 & 0.39 \\
\hline cardiovascular disease history(\%) & $3(5.17)$ & 6(13.95) & 2.34 & 0.12 \\
\hline MAP (mmHg) & $105.17 \pm 17.69$ & $110.93 \pm 17.24$ & -1.63 & 0.10 \\
\hline Net UF (ml/day) & & & -1.02 & 0.30 \\
\hline Median & 212.50 & 270.00 & & \\
\hline Interquartile range & $-50.00,712.50$ & $70.00,720$ & & \\
\hline Urine output (ml/day) & & & -1.32 & 0.18 \\
\hline Median & 600.00 & 500.00 & & \\
\hline Interquartile range & $200.00,1325.00$ & $20.00,1000.00$ & & \\
\hline Hemoglobin $(\mathrm{g} / \mathrm{L})$ & $104.17 \pm 24.25$ & $97.12 \pm 18.20$ & 1.66 & 0.10 \\
\hline Albumin (g/L) & $36.18 \pm 4.84$ & $31.09 \pm 5.70$ & 4.83 & $<0.01^{*}$ \\
\hline Total cholesterol (mmol/L) & $5.18 \pm 1.21$ & $4.64 \pm 1.26$ & 2.18 & $0.03^{*}$ \\
\hline Triglycerides (mmol/L) & $1.78 \pm 0.95$ & $1.59 \pm 1.02$ & 0.96 & 0.33 \\
\hline Calcium (mmol/L) & $2.10 \pm 0.24$ & $2.01 \pm 0.23$ & 1.80 & 0.07 \\
\hline Phosphorus (mmol/L) & $1.60 \pm 0.37$ & $1.37 \pm 0.49$ & 2.64 & $0.01^{*}$ \\
\hline Intact parathyroid hormone (pg/ml) & $434.62 \pm 328.77$ & $298.26 \pm 240.22$ & 2.30 & $0.02^{*}$ \\
\hline $\mathrm{rGFR}\left(\mathrm{ml} / \mathrm{min} / 1.73 \mathrm{~m}^{2}\right)$ & $5.67 \pm 2.68$ & $6.29 \pm 2.96$ & -1.09 & 0.27 \\
\hline $\mathrm{Kt} / \mathrm{N}$ & $2.11 \pm 0.46$ & $1.95 \pm 0.42$ & 1.71 & 0.09 \\
\hline $\mathrm{hsCRP}(\mathrm{mg} / \mathrm{dl})$ & & & -2.90 & $<0.01^{*}$ \\
\hline Median & 1.19 & 4.68 & & \\
\hline Interquartile range & $0.61,3.53$ & $0.66,12.04$ & & \\
\hline WBC $\left({ }^{*} 10 \wedge 3 / \mathrm{mm}^{3}\right)$ & $6.47 \pm 1.71$ & $6.42 \pm 2.11$ & 0.13 & 0.89 \\
\hline Neutrophil $\left({ }^{*} 10 \wedge 3 / \mathrm{mm}^{3}\right)$ & $4.32 \pm 1.35$ & $4.41 \pm 1.71$ & -0.30 & 0.76 \\
\hline Lymphocyte $\left({ }^{*} 10 \wedge 3 / \mathrm{mm}^{3}\right)$ & $1.45 \pm 0.43$ & $1.24 \pm 0.42$ & 2.45 & $0.01^{*}$ \\
\hline NLR & $3.17 \pm 1.20$ & $3.89 \pm 1.92$ & -2.15 & $0.03^{*}$ \\
\hline $\mathrm{baPWV}(\mathrm{cm} / \mathrm{s})$ & $1506.21 \pm 188.69$ & $2284.45 \pm 508.79$ & -9.55 & $<0.01^{*}$ \\
\hline
\end{tabular}

MAP mean arterial pressure, UF ultrafiltation, GFR glomerular filtration rate, $h S C R P$ high-sensitive C-reactive protein, WBC white blood count, baPWV brachial-ankle pulse wave velocity, NLR neutrophil-lymphocyte ratio; $p<0.05$ 
NLR was calculated as a ratio of neutrophil-tolymphocyte in peripheral blood. Mean arterial pressure (MAP) was calculated as $1 / 3$ systolic blood pressure plus $2 / 3$ diastolic blood pressure. Body mass index (BMI) was calculated as weight $(\mathrm{kg})$ divided by the square of height $\left(\mathrm{m}^{2}\right)$. Residual renal function (RRF) was assessed by calculating the glomerular filtration rate (GFR) using the Chronic Kidney Disease Epidemiology Collaboration equation. Adequacy of dialysis (total Kt/V) was measured using PD Adequest software.

\section{Measurement of the brachial-ankle pulse wave velocity (baPWV)}

baPWV was measured according to the method in a previous study [9], measurement of baPWV was performed after a 15-min rest in a supine position in a quiet and temperature-controlled room. The baPWV was measured based on regular methods using an Omron waveform analyzer (BP-203RPE III; Omron Co. Ltd, Dalian, China). The pulse wave velocity between the bilateral brachial and ankle artery was measured by placing both the arm and ankle in cuffs into which an oscillometric sensor was implanted. All the measurement were completed by the same doctor.

\section{Statistical analysis}

All statistical analyses were performed with SPSS software, version 19.0 (SPSS Inc.USA). A $p$-value $<0.05$ was considered statistically significant. The results were expressed as frequencies and percentages for categorical variables, means with standard deviation for normally distributed continuous variables, and median values (interquartile ranges) for non-normally distributed continuous variables, respectively. Student's $t$-test for independent samples was used for normally distributed $c$ ontinuous variables. Comparisons of non-normally distributed continuous variables were performed using the Mann-Whitney $U$-test. For categorical variables, the chisquare test was used. Correlations of baPWV with NRL and other variables were assessed using Pearson's or Spearman's correlation analysis. Univariate and multiple linear regression analyses were performed to identify the predictors of baPWV. Variables found to be statistically significant in univariate analyses were entered into multivariate lineal regression model.

\section{Results}

The demographic and biochemical characteristics of 101 subjects, according to baPWV levels are shown in Table 1. There were 58 patients in the low baPWV group and 43 patients in the high group. The raw data of the study can be seen in more detail (see Additional file 1). There were 100 patients treated with CAPD modality and one patient treated with APD modality. The primary etiology of end stage renal disease (ESRD) was chronic glomerulonephritis in both groups. There were significantly more patients with diabetes mellitus in the high baPWV group compared to the low baPWV group $\left(x^{2}=\right.$ $11.63, p<0.01)$.The number of the patients with cardiovascular diseases and current smoking status were similar between both groups. There were no differences between the groups in term of the following parameters: BMI, peritoneal dialysis duration, ultrafiltration, urine output, hemoglobin and triglyceride. Levels of albumin, total cholesterol, phosphorous, iPTH in the low group were significantly lower than those in the high group $(p<0.01, p=0.03, p=0.01, \quad p=0.02$, respectively). However, age and hsCRP were significantly higher in the low group than that in the high group (both $p<0.01$ ). There were slight differences in MAP, calcium, and Kt/V between groups, without reaching statistical significance ( $p=0.10, p=0.07, p=0.09$, respectively). In sub-analysis, average of baPWV was higher in patients with AVD history than those without AVD history $(\mathrm{t}=-2.99, p<0.01)$

When hematologic parameters were analyzed, it was observed that white blood cell (WBC) count and

Table 2 Correlation analysis for variables and baPWW

\begin{tabular}{|c|c|c|}
\hline Variables & $r$ & $p$-value \\
\hline Age (years) & 0.47 & $<0.01^{*}$ \\
\hline Body mass index $\left(\mathrm{kg} / \mathrm{m}^{2}\right)$ & -0.11 & 0.25 \\
\hline Peritoneal dialysis duration (months) & -0.01 & 0.84 \\
\hline Diabetes & 0.25 & $0.01^{*}$ \\
\hline current smoking status & 0.23 & $0.01^{*}$ \\
\hline atherosclerotic vascular disease history & 0.24 & $0.01^{*}$ \\
\hline MAP (mmHg) & 0.17 & 0.08 \\
\hline Net UF (ml/day) & 0.15 & 0.13 \\
\hline Urine output (ml/day) & 0.15 & 0.11 \\
\hline Hemoglobin (g/L) & -0.15 & 0.12 \\
\hline Albumin (g/L) & -0.32 & $<0.01^{*}$ \\
\hline Total cholesterol (mmol/L) & -0.12 & 0.22 \\
\hline Triglycerides (mmol/L) & -0.09 & 0.34 \\
\hline Calcium (mmol/L) & -0.03 & 0.70 \\
\hline Phosphorus (mmol/L) & -0.28 & $<0.01^{*}$ \\
\hline Intact parathyroid hormone (pg/ml) & -0.25 & $0.01^{*}$ \\
\hline $\mathrm{rGFR}\left(\mathrm{ml} / \mathrm{min} / 1.73 \mathrm{~m}^{2}\right)$ & 0.14 & 0.13 \\
\hline $\mathrm{Kt} / \mathrm{V}$ & -0.15 & 0.12 \\
\hline hsCRP(mg/dl) & 0.25 & $0.01^{*}$ \\
\hline WBC $\left({ }^{*} 10 \wedge 3 / \mathrm{mm}^{3}\right)$ & 0.16 & 0.09 \\
\hline Neutrophil $\left({ }^{*} 10 \wedge 3 / \mathrm{mm}^{3}\right)$ & 0.24 & $0.01^{*}$ \\
\hline Lymphocyte $\left(^{*} 10 \wedge 3 / \mathrm{mm}^{3}\right)$ & -0.23 & $0.01^{*}$ \\
\hline NLR & 0.43 & $<0.01^{*}$ \\
\hline
\end{tabular}

MAP mean arterial pressure, UF ultrafiltation, GFR glomerular filtration rate, hSCRP high-sensitive C-reactive protein, WBC white blood count, baPWV brachial-ankle pulse wave velocity, NLR neutrophil-lymphocyte ratio;* $p<0.05$ 
neutrophil counts were similar between groups ( $p=0.89$, $p=0.76$, respectively). However, the lymphocyte count was significantly higher in low group compared to in high group $(p=0.01)$. NLR was significantly lower in the low group than that in the high group $(p=0.03)$ (Table 1).

Correlations between baPWV and parameters in patients with PD are shown in Table 2. According to the results, there were positive correlations of baPWV and neutrophil count $(r=0.24, p=0.01)$ and NRL $(r=0.43, p<0.01)$, and there was a negative correlation between baPWV and lymphocyte count $(r=-0.23, P=0.01)$. In addition, albumin, phosphorous and iPTH showed negative correlations with baPWV $(r=-0.32, P<0.01 ; r=-0.28, P<0.01 ; r=-0.25, P=$ $0.01)$. Age and hsCRP showed positive correlation with baPWV $(r=0.47, p<0.01 ; r=0.25, p=0.01)$. Correlation between baPWV and MAP approached statistical significance $(r=0.17, p=0.08)$.

Variables found to be statistically significant in univariate analyses were entered into multivariate lineal regression model, age, diabetes, current smoking status, AVD history, albumin,phosphorus, iPTH and NLR. In multivariate analysis, NLR independently correlated with baPWV in patients with $\mathrm{PD}(\beta=0.33$, $p<0.01$ ) (Table 3).

\section{Discussion}

This study revealed that NLR was higher in the low baPWV group than that in the high baPWV group. Moreover, it showed that NRL, a new inflammatory marker, was associated with arterial stiffness in patients with PD. Multiple linear regression analysis showed that NLR was an independent factor for increased baPWV.

Compared to general population, CKD patients have increased arterial stiffness [10]. Arterial stiffness plays a key role in the pathophysiology of the cardiovascular disease. baPWV is a commonly applied non-invasive technique to measure arterial stiffness [11]. Accumulated evidence has indicated that baPWV is an independent predictor of cardiovascular outcomes in patients with CKD $[12,13]$. In our study the average level of baPWV was higher in patients with AVD history than those without AVD history. Recent studies have emphasized the importance of inflammation in the pathogenesis of arterial stiffness. Duprez et al. have demonstrated a significant correlation between arterial stiffness and hsCRP [14]. Also, previous studies suggested that reduction in inflammation can decrease arterial stiffness [15].

Recent studies have shown that a potential association between NLR and baPWV in patients with type 1 diabetes [16], osteoporosis [17] or psoriasis [18]. Our study was also in accordance with these studies. The study is the first to show that serum NLR was positively and independently correlated with baPWV in adult PD patients, even after adjustment for various confounders. Moreover, NLR was superior to hsCRP in association with PWV. These results demonstrated that inflammation plays a crucial role in the development of arterial stiffness among patients with PD [19].

The NLR reflects both neutrophil and lymphocyte counts. Our results showed that neutrophil counts were

Table 3 Univariate and multivariate associates of baPW in patients with peritoneal dialysis

\begin{tabular}{|c|c|c|c|c|c|c|}
\hline \multirow[t]{3}{*}{ Variables } & \multicolumn{6}{|l|}{ baPWV } \\
\hline & \multicolumn{3}{|l|}{ Univariable } & \multicolumn{3}{|l|}{ Multivariate } \\
\hline & $\beta$ coefficient & $\mathrm{t}$ & $P$-value & $\beta$ coefficient & $\mathrm{t}$ & $P$-value \\
\hline Age (year) & 0.47 & 5.38 & $<0.01^{*}$ & 0.25 & 2.61 & $<0.01^{*}$ \\
\hline Body mass index $\left(\mathrm{kg} / \mathrm{m}^{2}\right)$ & -0.11 & -1.14 & 0.26 & - & - & - \\
\hline Diabetes (\%) & 0.23 & 2.44 & $0.01^{*}$ & 0.04 & 0.53 & 0.59 \\
\hline current smoking status (\%) & 0.25 & 2.55 & $0.01^{*}$ & -0.18 & -1.00 & 0.31 \\
\hline atherosclerotic vascular disease history (\%) & 0.28 & 2.99 & $<0.01^{*}$ & 0.32 & 1.75 & 0.08 \\
\hline MAP (mmHg) & -0.17 & 1.72 & 0.08 & - & - & - \\
\hline Albumin $(\mathrm{g} / \mathrm{L})$ & -0.31 & -3.34 & $<0.01^{*}$ & -0.17 & -1.99 & $0.04^{*}$ \\
\hline Calcium (mmol/L) & -0.03 & -0.37 & 0.70 & - & - & - \\
\hline Phosphorus (mmol/L) & -0.28 & -2.98 & $<0.01^{*}$ & -0.12 & -0.14 & 0.16 \\
\hline iPTH (pg/ml) & -0.25 & -2.62 & $0.01^{*}$ & -0.43 & -0.49 & 0.66 \\
\hline $\mathrm{rGFR}\left(\mathrm{ml} / \mathrm{min} / 1.73 \mathrm{~m}^{2}\right)$ & 0.15 & 1.49 & 0.13 & - & - & - \\
\hline $\mathrm{Kt} / \mathrm{V}$ & -0.15 & -1.57 & 0.12 & - & - & - \\
\hline $\mathrm{hsCRP}(\mathrm{mg} / \mathrm{dl})$ & 0.19 & 1.96 & 0.05 & - & - & - \\
\hline NLR & 0.43 & 4.74 & $<0.01^{*}$ & 0.33 & 3.91 & $<0.01^{*}$ \\
\hline
\end{tabular}

$M A P$ mean arterial pressure, UF ultrafiltation, GFR glomerular filtration rate, $h S C R P$ high-sensitive C-reactive protein, WBC white blood count, baPWV brachial-ankle pulse wave velocity, NLR neutrophil-lymphocyte ratio; $p<0.05$ 
similar in both groups with high or low baPWV, but lymphocyte counts showed significant decrease and NLR showed significant increase in the high group compared to the low baPWV. Thus, increased NLR are more likely due to decreased absolute lymphocyte counts rather than increased neutrophil counts in our study. This is consistent with the study by Solak et al. [20]. The previous study has shown that NLR is superior to these two leukocyte parameters, the most powerful simple leukocyte count indicators [21]. Compared to these two count indicators, it is more stable and less affected by acute conditions which change one of the individual cell counts.

Patients with PD were in a low graded chronic inflammation state. WBC subtypes play the role in different ways to promote atherosclerosis. Increasing evidence has shown that neutrophil directly acts as a mediator of tissue destruction to the arterial wall in inflammation conditions [22]. Lymphopenia may be caused by lymphocyte apoptosis in atherosclerotic lesions, which gradually increases with atherosclerotic burden [23]. Moreover, several studies showed a relationship between low lymphocyte count and malnutrition [24, 25]. Thus, the combination of elevated neutrophils (nonspecific inflammation) and decreased levels of lymphocytes (regulatory component) into a single composite marker may provide additional information to interpret the pathogenesis of arterial stiffness [26]. However, there is an ongoing debate whether NLR is merely a surrogate marker of understanding inflammation, or whether it confers an increased risk per se [20]. More researches are warranted to investigate further.

There are three main limitations in this study. Firstly, our study population is just from a single center, which cannot represent the general population. Secondly, due to the nature of this cross-section study design, we cannot draw a cause-and-effect relationship. Thirdly, as there is no healthy control, it is hard to gain the comparison of baPWV and NLR between patients with PD and healthy persons.

\section{Conclusion}

In conclusion, our results showed that NLR was independently associated with arterial stiffness. It is stable and ready to evaluate NLR and baPWV to confirm inflammation and subclinical atherosclerosis in patients with PD. However, further prospective studies are needed to confirm a cause-and-effect relationship between NLR and baPWV and to investigate whether antiinflammatory treatment could improve arterial stiffness in patients with PD.

\section{Additional file}

Additional file 1: Raw data of the study. (XLS $46 \mathrm{~kb}$ )

\section{Abbreviations}

AVD: Atherosclerotic vascular disease; baPWV: Brachial-ankle pulse wave velocity; BMI: Body mass index; CKD: Chronic kidney disease; ESRD: End stage renal disease; GFR: Glomerular filtration ratev; hsCRP: Highly sensitive Creactive protein; iPTH: Intact parathyroid hormone; MAP: Mean arterial pressure; MIAC: Malnutrition-inflammation-atherosclerosis and calcification; NLR: Neutrophil-lymphocyte ratio; PD: Peritoneal dialysis; RRF: Residual renal function; WBC: White blood cell

\section{Acknowledgment}

The authors thank the staff in the Department of Nephrology in Ningbo No.2 Hospital, and our patients for their participation.

\section{Funding}

This work was supported by Ningbo social-development fund (No. 2015C50038) to LQ.

\section{Availability of data and materials}

All data underlying the findings are within the paper and the supporting information file (see Additional file 1).

\section{Authors' contributions}

CKD: conceived, designed and performed the experiment as well as wrote the manuscript. LQ: conceived, designed the experiment, meanwhile revised the manuscript. ZBX: measured baPWV. HLN: measured baPWV. DZW: collected the data. WD: collected the data. WKY: collected the data. All the authors read and approved the manuscript.

\section{Competing interests}

The authors declare that they have no competing interests.

\section{Consent for publication}

Not applicable.

\section{Ethics approval and consent to participate}

This study was approved by the Ethics Committee of Ningbo No.2 Hospital (No. PJ-KY-NBEY-2015-002-01). Consent to participate was not required as this was a retrospective study, however, patients' information was anonymized and de-identified prior to analysis.

Received: 24 April 2016 Accepted: 9 November 2016

Published online: 24 November 2016

\section{References}

1. Cai KD, Luo Q, Dai ZW, Zhu BX, Fei JP, Xue CP, Wu D. Hypomagnesemia is associated with increased mortality among peritoneal dialysis patient. Plos One. 2016;11(3):e0152488.

2. Tamhane UU, Aneja S, Montgomery D, Rogers EK, Eagle KA, Gurm HS. Association between admission neutrophil to lymphocyte ratio and outcomes in patients with acute coronary syndrome. Am J Cardiol. 2008;102:653-7.

3. Turkmen K, Guney I, Yerlikaya FH, Tonbul HZ. The relationship between neutrophil-to-lymphocyte ratio and inflammation in end-stage renal disease patients. Ren Fail. 2012;34:155-9.

4. Karras A, Haymann JP, Bozec E, Metzger M, Jacquot C, Maruani G, Houillier P, Froissart $M$, Stengel B, Guardiola $P$, et al. Large artery stiffening and remodeling are independently associated with all-cause mortality and cardiovascular events in chronic kidney disease. Hypertension. 2012;60:1451-7.

5. Mancia G, De Backer G, Dominiczak A, Cifkova R, Fagard R, Germano G, Grassi G, Heagerty AM, Kjeldsen SE, Laurent S, et al. Guidelines for the management of arterial hypertension: the task force for the management of arterial hypertension of the european society of hypertension (esh) and of the european society of cardiology (esc). J Hypertens. 2007;25:1105-87.

6. Tanaka H, Munakata M, Kawano Y, Ohishi M, Shoji T, Sugawara J, Tomiyama H, Yamashina A, Yasuda H, Sawayama T, et al. Comparison between carotidfemoral and brachial-ankle pulse wave velocity as measures of arterial stiffness. J Hypertens. 2009;27:2022-7.

7. Tomiyana $\mathrm{H}$, Tanaka $\mathrm{H}$, Hashimoto $\mathrm{H}$, Matsumoto C, Odaira M, Yamada J, Yoshida M, Shiina K, Nagata M, Yamashina A. Arterial stiffness and declines in individuals with normal renal functional/early chronic kidney disease. Atherosclerosis. 2010;212:345-50. 
8. Xu L, Jiang CQ, Lam TH, Yue XJ, Cheng KK, Liu B, Jin YL, Zhang WS, Thomas GN. Brachial-Ankle pulse wave velocity and cardiovascular risk factors in non-diabetic and newly diagnosed diabetic Chinese:Guangzhou Biobank Cohort Study-CVD. Diabetes Metab Res Rev. 2010;26:133-9.

9. Luo Q, Wang LL, Gao YH. Association between serum 25-hydroxyvitamin D and arterial stiffness in non-dialysis-dependent CKD. Eur J Clin Nutr. 2016; 70(2):274-6.

10. Shinohara K, Shoji T, Tsujimoto Y, Kimoto E, Tahara H, Koyama H, Emoto M, Ishimura E, Miki T, Tabata T, et al. Arterial stiffness in predialysis patients with uremia. Kidney In. 2004;65(3):936-43.

11. Yamashina A, Tomiyama H, Takeda K, Tsuda H, Arai T, Hirose K, Koji Y, Hori $S$, Yamamoto Y. Validity, reproducibility, and clinical significance of noninvasive brachial-ankle pulse wave velocity measurement. Hypertens Res. 2002;25:359-64.

12. Morimoto $S$, Yurugi T, Aota Y, Sakuma T, Jo F, Nishikawa M, Iwasaka T, Maki K. Prognostic significance of ankle-brachial index, brachial-ankle pulse wave velocity, flow-mediated dilation, and nitroglycerin - mediated dilation in end-stage renal disease. Am J Nephrol. 2009;30:55-63.

13. Washida N, Wakino S, Hayashi K, Kuwahara T, Itoh H. Brachial-ankle pulse wave velocity predicts silent cerebrovascular diseases in patients with endstage renal diseases. J Atheroscler Thromb. 2010;17:165-72.

14. Duprez DA, Somasundaram PE, Sigurdsson G, Hoke L, Florea N, Cohn JN. Relationship between C-reactive protein and arterial stiffness in asymptomatic population. J Hum Hypertens. 2005;19:515-9.

15. Van Doornum S, McColl G, Wicks IP. Atorvastatin reduces arterial stiffness in patients with rheumatoid arthritis. Ann Rheum Dis. 2004;63:1571-5.

16. Ayhan H, Kasapkara HA, Aslan AN, Durmaz T, Keleş T, Akçay M, Akar Bayram N, Baştuğ S, Bilen E, Sarı C, et al. Relationship of neutrophil-to-lymphocyte ratio with aortic stiffness in type 1 diabetes mellitus. Canadian J Diabetes. 2015;39:317-21.

17. Yu XY, Li XS, Li Y, Liu TM, Wang RT. Neutropihl-lymphocyte ratio is associated with arterial stiffness in postmenopausal women with osteoporosis. Arch Gerontol Geriatr. 2015;61:76-80.

18. Sunbul M, Seckin D, Durmus E, Ozgen Z, Bozbay M, Bozbay A, Kivrak T, Oguz M, Sari I, Ergun T, et al. Assessment of arterial stiffness and cardiovascular hemodynamics by oscillometric method in psoriasis patients with normal cardiac functions. Heart Vessel. 2015;30:347-54.

19. Krzanowski M, Janda K, Dumnicka P, Dubiel M, Stompór M, Kuśnierz-Cabala B, Grodzicki T, Sułowicz W. Relationship between aortic pulse wave velocity, selected proinflammatory cytokines, and vascular calcification parameters in peritoneal dialysis patients. J Hypertens. 2014;32(1):142-8.

20. Solak Y, Yilmaz MI, Sonmez A, Saglam M, Cakir E, Unal HU, Gok M, Caglar K, Oguz $Y$, Yenicesu $M$, et al. Neutrophil to lymphocyte ratio independently predicts cardiovascular events in patients with chronic kidney disease. Clin Exp Nephrol. 2013;17:532-40

21. Horne BD, Anderson JL, John JM, Weaver A, Bair TL, Jensen KR, Renlund DG, Muhlestein JB. Which white blood cell subtypes predict increased cardiovascular risk? J Am Coll Cardiol. 2005:45:1638-43.

22. Baetta $R$, Corsini A. Role of polymorphonuclear neutrophils in atherosclerosis: current state and future perspectives. Atherosclerosis. 2010 210(1):1-13.

23. Nunez J, Sanchis J, Bodi V, Núñez E, Mainar L, Heatta AM, Husser O, Miñana $G$, Merlos P, Darmofal H, et al. Relationship between low lymphocyte count and major cardiac events in patients with acute chest pain, a nondiagnostic electrogram and normal troponin levels. Atherosclerosis. 2009; 206(1):251-7.

24. Mcmillan DC. Systemic inflammation, nutrition status and survival in patients with cancer. Curr Opin Clin Nutr Metab Care. 2009;12(3):223-6.

25. Battin DL, Ali S, Shahbaz AU, Massie JD, Munir A, Davis Jr RC, Newman KP, Weber KT. Hypoalbuminemia and lymphocytopenia in patients with decompensated biventricular failure. Am J Med Sci. 2010:339(1):31-5.

26. Acar G, Fidan S, Alnur Z, Turkday S, Avcl A, Alizade E, Alkan ME, Tabakci ON, Tanboğa IH, Esen AM. Relationship of neutrophil-lymphocyte ratio with the presence, severity, and extent of coronary atherosclerosis detected by coronary computed tomography angiography. Angiology. 2015;66(2):174-9.

\section{Submit your next manuscript to BioMed Central and we will help you at every step:}

- We accept pre-submission inquiries

- Our selector tool helps you to find the most relevant journal

- We provide round the clock customer support

- Convenient online submission

- Thorough peer review

- Inclusion in PubMed and all major indexing services

- Maximum visibility for your research

Submit your manuscript at www.biomedcentral.com/submit
() BioMed Central 